\title{
ISOPHOTOMETRY OF COMET TAGO-SATO-KOSAKA
}

\author{
C. K. Kumar and Rita J. Southall
}

\begin{tabular}{|c|c|c|c|c|c|}
\hline \multicolumn{6}{|c|}{ ABSTRACT } \\
\hline \multicolumn{6}{|c|}{$\begin{array}{l}\text { Narrow-band filler photographs of comet TSK were taken in the light of } \mathrm{C}_{2}, \mathrm{C} \\
\text { by Rahe McCracken and Donn, have been analysed in terms of Haser's model of the } \\
\text { The isophotes obtained from these photographs were corrected for sky background } \\
\text { isophotes were circularly symmetric. Radial intensity profiles were obtained along } \\
\text { ward, antisun and the two perpendicular directions. In each case, these profiles wer } \\
\text { same within the experimental errors in intensity }( \pm 5 \%) \text {. }\end{array}$} \\
\hline \multirow{2}{*}{\multicolumn{6}{|c|}{$\begin{array}{l}\text { Theoretical curves based on Haser's model were computed for different combi } \\
\text { of the parameters } \beta_{0} \text { and } \beta_{1} \text { where } 1 / \beta_{0} \text { is the scale length for the decay of the unot } \\
\text { parent molecule and } 1 / \beta_{1} \text { the corresponding quantity for the observed daughter mo } \\
\text { comparison of the theoretical and observed intensity profiles, yielded our best estim } \\
\beta_{0} \text { and } \beta_{1} \text { for the different molecules. These are listed in Table } 1 \text {. In some cases, w } \\
\text { able to obtain lower limits only to the ratio } \beta_{0} / \beta_{1} \text { because the isophotes do not go } \\
\text { enough, a limitation caused by the sky background. It is to be noted that the same } \\
\text { the parameters were obtained from observations on different dates indicating no un } \\
\text { activity in the comet in the intervening period. We believe ours is the first result ev } \\
\text { tained for } C_{3} \text {. } \\
\text { In Table } 2 \text {, we summarize all the results for } C_{2} \text { and } \mathrm{CN} \text { in different comets. It } \\
\text { that the scale lengths differ between comets, a result which cannot be attributed to } \\
\text { ent helio-centric distances alone. }\end{array}$}} \\
\hline & & & & & \\
\hline \multicolumn{6}{|c|}{$\begin{array}{c}\text { Table } 1 \\
\mathrm{C}_{2} \text { and CN Scale Lengths - Comet TSK }\end{array}$} \\
\hline $\begin{array}{l}\text { Observation } \\
\text { Date } \\
\text { UT }\end{array}$ & $\begin{array}{l}\text { Molecule } \\
\text { Wavelength } \\
\quad \AA\end{array}$ & $\begin{array}{l}\beta_{0} \\
\times 10^{5} \\
\mathrm{KM}^{-1}\end{array}$ & $\beta_{0} / \beta_{1}$ & $\begin{array}{r}\text { Decay } \\
\text { Uni } \\
\text { (Parent) }\end{array}$ & $\begin{array}{l}\text { Length in } \\
10^{4} \mathrm{KM} \\
\text { (Daughter) }\end{array}$ \\
\hline $2 / 11.99$ & $\mathrm{CN}-3884$ & $5 .-6$ & $2.5-4$. & $1.67-2$ & 4.175-8. \\
\hline $2 / 13.026$ & $\mathrm{CN}-3884$ & 5.-6. & $2.5-4$. & $1.67-2$. & 4.175-8. \\
\hline $2 / 14.026$ & $\mathrm{CN}-3884$ & $5 .-6$. & $2.5-4$ & $1.67-2$ & 4.175-8. \\
\hline $2 / 12.003$ & $C_{2}-5172$ & 4.0 . & 4.-6. & 2.5. & $10 .-15$ \\
\hline $2 / 12.015$ & $C_{2}-5172$ & 4.0 . & 4.-6. & 2.5 . & $10 .-15$ \\
\hline $2 / 13.013$ & $C_{2}-5172$ & 4.0 . & 4.-6. & 2.5 . & $10 .-15$. \\
\hline $2 / 14.018$ & $C_{2}-5172$ & 4.0 & 4.-6. & 2.5 & $10 .-15$ \\
\hline $2 / 12.026$ & $C_{2}-4738$ & 4.0 & 4.-6. & 2.5 & $10 .-15$ \\
\hline $2 / 12.047$ & $C_{3}-4063$ & $12 .-13$. & 2. & $76-.83$ & $1.54-1.67$ \\
\hline
\end{tabular}


Table 2

Comparison of $\mathrm{CN}$ and $\mathrm{C}_{2}$ Scale Lengths

\begin{tabular}{|c|c|c|c|c|c|}
\hline Reference & $\begin{array}{c}\text { Molecule } \\
\text { Wavelength } \\
\AA\end{array}$ & $\begin{array}{c}\text { Comet } \\
\text { Helio- } \\
\text { centric } \\
\text { Distance } \\
\text { A.U. }\end{array}$ & $\begin{array}{c}\text { Observation } \\
\text { Date } \\
\text { UT }\end{array}$ & $\begin{array}{r}\text { Sc } \\
\text { in Uni } \\
\text { Parent } \\
1 / \beta_{0}\end{array}$ & $\begin{array}{c}10^{4} \mathrm{KM} \\
\text { Daughter } \\
1 / \beta_{1}\end{array}$ \\
\hline $\begin{array}{l}\text { This } \\
\text { Analysis }\end{array}$ & CN-3884 & $\begin{array}{l}\text { TSK } \\
(1.239- \\
1.275)\end{array}$ & $\begin{array}{c}2 / 11.99- \\
2 / 14.026 \\
1970\end{array}$ & $1.67-2$ & $4.175-8$ \\
\hline $\begin{array}{l}\text { Borra \& } \\
\text { Wehlau } \\
\text { (1973) }\end{array}$ & CN-3878 & $\begin{array}{l}\text { TSK } \\
(1.095)\end{array}$ & $\begin{array}{c}2 / 4.0 \\
1970\end{array}$ & 2.5 & 20.0 \\
\hline $\begin{array}{l}\text { Delsemme \& } \\
\text { Moreau } \\
\text { (1973) }\end{array}$ & $\mathrm{CN}$ & $\begin{array}{l}\text { Bennett } \\
(1.0)\end{array}$ & $\begin{array}{l}3 / 30- \\
5 / 7 \\
1970\end{array}$ & 5.01 & 14.1 \\
\hline $\begin{array}{l}\text { This } \\
\text { Analysis }\end{array}$ & $\begin{array}{r}C_{2}-5172 \\
-4738\end{array}$ & $\begin{array}{l}\text { TSK } \\
(1.239 \\
1.275)\end{array}$ & $\begin{array}{c}2 / 12.033- \\
2 / 14.018 \\
1970\end{array}$ & 2.5 & $10-15$ \\
\hline $\begin{array}{l}\text { Delsemme \& } \\
\text { Moreau } \\
\text { (1973) }\end{array}$ & $\mathrm{C}_{2}$ & $\begin{array}{l}\text { Bennett } \\
(1.0)\end{array}$ & $\begin{array}{l}3 / 30- \\
5 / 7 \\
1970\end{array}$ & 2.0 & 6.31 \\
\hline $\begin{array}{l}\text { Dewey \& } \\
\text { Miller } \\
\text { (1966) }\end{array}$ & $C_{2}-5165$ & $\begin{array}{l}\text { Seki } \\
(.935)\end{array}$ & $\begin{array}{r}11 / 11.4 \\
1961\end{array}$ & 4.57 & 8.32 \\
\hline $\begin{array}{l}\text { O’Dell \& } \\
\text { Osterbrock } \\
\text { (1962) }\end{array}$ & $C_{2}-4737$ & $\begin{array}{l}\text { Seki } \\
(.922 \\
.935)\end{array}$ & $\begin{array}{c}11 / 10.4 \& \\
11 / 11.4 \\
1961\end{array}$ & 1.42 & 8.51 \\
\hline $\begin{array}{l}\text { Dewey \& } \\
\text { Miller } \\
(1966)\end{array}$ & $\mathrm{C}_{2}$ & $\begin{array}{l}1955 \mathrm{e}^{*} \\
1955 \mathrm{~g}^{*} \\
1959 \mathrm{k}^{* *}\end{array}$ & & 1.167 & 9.33 \\
\hline
\end{tabular}

*Observations by Schmidt and van Woerden (1957)

**Observations by Miller (1961) 